\title{
Adhesive Properties Study of f-SWCNTs:P3OT Nanocomposite Thin Film
}

\author{
J. Alzanganawee ${ }^{1,2}$, O. Brincoveanu ${ }^{1,3}$, R. Mesterca $^{1}$, D. Balan ${ }^{1,3}$, \\ A. Apaz ${ }^{1,3}$ M. Enachescu ${ }^{1,4}$ \\ ${ }^{1}$ Center for Surface Science and Nanotechnology (CSSNT), University Politehnica of Bucharest, Romania \\ ${ }^{2}$ Physics Department, College of Science, University of Diyala, Iraq \\ ${ }^{3}$ University of Bucharest, Faculty of Physics, Bucharest, Romania \\ ${ }^{4}$ Academy of Romanian Scientists, Bucharest, Romania \\ marius.enachescu@upb.ro
}

\begin{abstract}
Topography and 'pull off' curves are two of the most important among the many available AFM modes, which are enabling the morphological and mechanical properties investigations of the surfaces. It is known that nanocomposites synthesizing has attracted the worldwide scientists' interest, due to the enlarged properties of these materials. There are few studies on P3OT:SWCNTs nanocomposites used in OPVs when compared to fullerene derivatives and even fewer focused on mechanical properties, as the main research direction was to increase the efficiency. In this paper, preparation and characterization of the P3OT pure polymer and 12\% f-SWCNTs:P3OT nanocomposite, respectively, were reported. The experimental results showed the noticeable effect of the carbon nanotubes addition into the polymer matrix. The surface roughness of the nanocomposite increased due to the presence of the $f-$ SWCNTs, while the adhesion force values decreased. The adhesion force variation distribution becomes more uniform, with the carbon nanotubes addition into the host.
\end{abstract}

Keywords: topography, adhesion, P3OT, f-SWCNTs, nanocomposite

\section{Introduction}

With the development of the Atomic Force Microscopy (AFM) besides surface morphological analysis the investigations of various properties over different material surfaces at the nanoscale level, such as elastic and adhesion properties, became easily achievable $\left[{ }^{1}\right]$.

Adhesion forces can describe the interconnection at interfaces between single or multi-layer systems, and it may be either due to chemical bonding or mechanical interlocking interactions $\left[{ }^{2}\right]$. It is a known fact that the mechanism behind adhesion differs from macroscopic to microscopic level $\left[{ }^{3}\right]$. At molecular level there is no need for various adhesives or joints to bond two components, rather the objects spontaneously attach to each other and they are taking apart with difficulty. This type of adhesion is called "pre-mature adhesion" and the bond between two objects cannot be broken without the use of force or through some kind of surface contamination $\left[{ }^{3}\right]$.

In this paper we report the study of topography, morphology and the adhesive properties on P3OT and 12 wt. \% f-SWCNTs P3OT thin film samples, respectively. In the organic optoelectronic industry, there is an underlying focus on poly(3-octylthiophene-2,5-diyl (P3OT) and poly(3-hexylthiophene-2,5-diyl) (P3HT) $\pi$-conjugated polymers $\left[{ }^{4}\right]$. A special interest has been given to conjugated polymer:fullerene derivatives as the active layer in OPVs in contrast to polymer:SWCNTs hybrid structures. The main focus within the research directed towards OPVs has been on the enhancing the solar cell's efficiency to enable their large scale use, while studies on mechanical properties, such as adhesion and cohesion are scarce, even though they play a vital role in device reliability and stability $\left[{ }^{5},{ }^{6},{ }^{7}\right]$. The nanocomposite thin film consisting of the polymer reinforced with carbon nanotubes exhibits new mechanical and 
morphological properties $\left[{ }^{8},{ }^{9}\right]$. In the analysed samples, SWCNTs in the polymer matrix could be observed in the acquired topography image and were found to have an influence on both the uniformity of distribution and adhesion force values.

\section{Experimental}

\subsection{Sample preparation}

The materials used for sample preparation were commercially available P3OT and SWCNTs. P3OT polymer was used as-purchased from Sigma Aldrich and did not require further processing. SWCNTs were acquired from NanoIntegris Inc, with a purity of approximately $70 \%$, a diameter ranging from 0.9 to $1.7 \mathrm{~nm}$ and lengths in the 0.3 4 um range. The nanotubes were further processed using a functionalization procedure with $6 \mathrm{M}$ nitric acid solution to yield f-SWCNTs.

A three-step procedure was used to obtain the nanocomposites mixture, using P3OT as the polymer matrix and f-SWCNTs (12 wt.\%) as the reinforcing phase. Firstly, the nanotubes were dispersed into chloroform solvent at room temperature for 30 minutes with the aid of a high ultrasonic tip, with an energy about 15000 Joule to ensure a better incorporation of the filler into the matrix in the next step. Afterwards, the resulted dispersion was blended with the polymer and finally the nanocomposite solution was sonicated for $1 \mathrm{~h}$ at $45-50^{\circ} \mathrm{C}$ to increase the homogeneity of the mixture.

A pre-deposition cleaning of the glass substrates was performed with acetone, isopropyl alcohol and deionized water for 15 minutes using an ultrasound bath. Two thin films were spincoated for $30 \mathrm{sec}$, at $1000 \mathrm{rpm}$, in ambient conditions on the cleaned substrate using the aspurchased P3OT solution and the nanocomposites mixture.

\subsection{Sample characterization}

Surface topography and adhesion properties of the prepared thin films were investigated using a multi-mode commercial atomic force microscope (NTEGRA - NTMDT), in ambient conditions. For these measurements, carried out in AFM contact mode, commercially available standard cantilever chips with a $0.26 \mathrm{~N} / \mathrm{m}$ stiffness and monocrystalline silicon tips with radius of approximately $10 \mathrm{~nm}$ were used.

The surface roughness and Skewness parameters were determined from the acquired topographic images using the equations (1) and (2), with the help of an image processing software.

$$
\begin{gathered}
\mathbf{R a}=\sum_{i=0}^{N} \frac{\mathbf{h}_{\mathbf{i}}}{N} . \\
\mathbf{R}_{\text {Skew }}=\frac{1}{\mathrm{NR}_{\mathbf{q}}^{3}} \sum_{\mathrm{i}=1}^{\mathrm{N}}\left(\mathbf{h}_{\mathrm{i}}-\overline{\mathbf{h}}\right)^{3}
\end{gathered}
$$

The adhesion properties measurements, performed in AFM spectroscopy for all the samples, were determined from the 'pull off' region of the force-distance curves, applying a constant force between the tip and the sample. The adhesion force $\mathrm{F}(\mathrm{nN})$ between the tip and the sample was calculated using the equation ' $\mathrm{F}=\mathrm{k} \mathrm{x}$ $\Delta d$ ', where $\mathrm{k}$ is the spring constant of the cantilever and $\Delta d$ is the deflection distance. For this purpose, adhesion maps consisting of 400 curves on a 10x10 um size were acquired.

The internal structure of the Nano composite was analyzed using a Hitachi HD 2700 High Resolution Scanning Transmission Electron Microscope (HR-STEM).

\section{Results and discussion}

The as-prepared nanocomposite thin layers are characterized by their 3D imaged topographies, displayed in figure 1,2 . The noticeable uniformity of the pure P3OT polymer observed in its acquired topography image is confirmed also by its revealed roughness $(\mathrm{Ra})$ of $0.99 \mathrm{~nm}$, while the high surface roughness parameter value $(31.89 \mathrm{~nm})$ determined for the $12 \%$ f-SWCNTs:P3OT sample is clearly influenced by the presence of the carbon nanotubes into the polymer matrix, which leads to the formation of broad valleys and hills seen in the topography image of the nanocomposite.

Due to the high attraction between them caused by the Van der Walls forces $\left[{ }^{10}\right]$, the carbon nanotubes are matched into bundles, as they appear in the $12 \%$ f-SWCNTs:P3OT sample's topography image.

On the other hand, the calculated Skewness parameter values for both investigated samples, 
pure polymer and nanocomposite, of about 0.7 and 0.9 , respectively, reveal the comparable positive asymmetries of the heights and depths distributions over the surfaces, which can be explained if we assume a homogenous distribution of the carbon nanotubes into the host. A positive value for the Skewness parameter translates into the prevalence of the peaks present in the topography images $\left[{ }^{11}\right]$.

Table 1. Morphological parameters

\begin{tabular}{cccc} 
Sample & $\begin{array}{c}\mathrm{Ra} \\
(\mathrm{nm})\end{array}$ & Rskew & $\begin{array}{c}\overline{F_{a d}} \\
(\mathrm{nN})\end{array}$ \\
\hline P3OT & 0.99 & 0.7 & 16.1 \\
\hline 12\% f-SWCNTs:P3OT & 31.89 & 0.9 & 14.4
\end{tabular}

$20 \mathrm{~nm}$

$0 \mathrm{~nm}$

Fig. $13 \mathrm{D}$ AFM topography image of the P3OT pure polymer

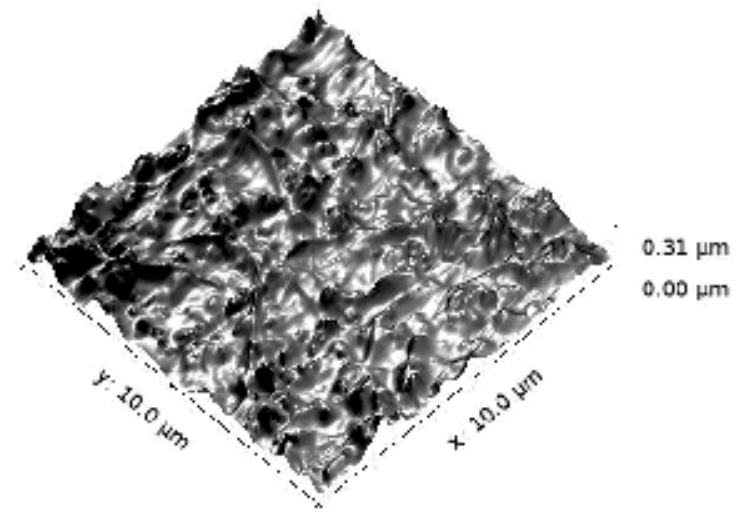

Fig. 2 3D AFM topography image of the fSWCNTs:P3OT nanocomposite

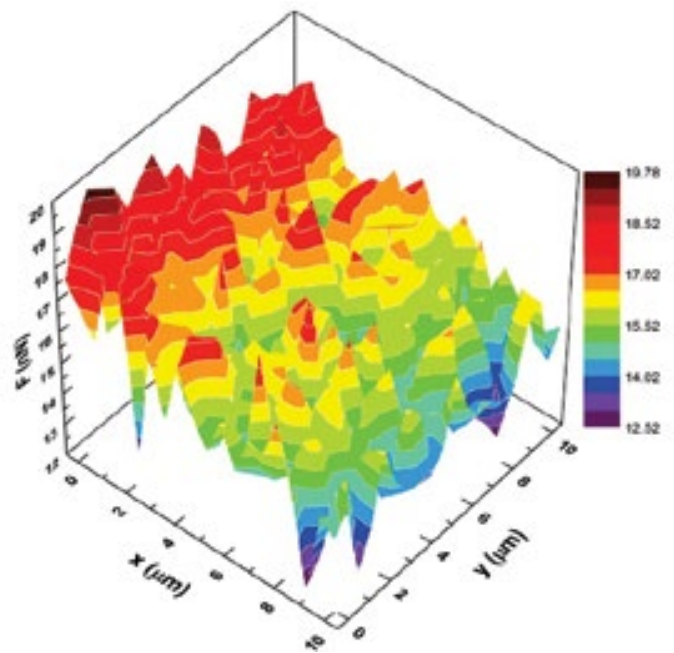

Fig. 3 3D Adhesion force variation map for the P3OT pure polymer

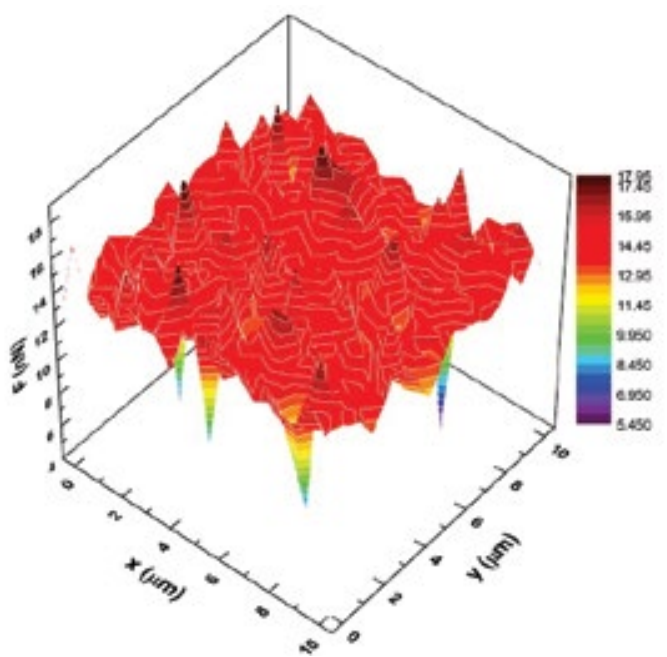

Fig. 4 3D Adhesion force variation map for the fSWCNTs:P3OT nanocomposite

The changes of the surface properties $\left[{ }^{12}\right]$ with the carbon nanotubes addition into the polymer matrix are revealed by the imaged force distance measurements, illustrated in figure 3,4 . As previously was mentioned each adhesion map was plotted as an adhesion force values dependence of the $(\mathrm{x}, \mathrm{y})$ position of each from those 400 forcedistance curves acquired.

In the adhesion map obtained for the pure polymer adhesion force values, ranged from 12.5 to $19.8 \mathrm{nN}$, are grouped in large regions with approximately the same value, while the presence 
of the carbon nanotubes bundles into the host leads to a more uniform distribution of the adhesion force values over the nanocomposite surface.

An overview of the obtained adhesion force values for the analysed samples reveals the decisive role of the carbon nanotubes addition. The calculated mean adhesion values (tab. 1) for each map of about $16.1 \mathrm{nN}$ and $14.4 \mathrm{nN}$ for pure polymer and nanocomposite, respectively, reveal the changes of adhesive properties of the surfaces, as a result of carbon nanotubes addition.

The variation of adhesion force is dependent on the contact area (tip-sample interaction), so the increase of the surface roughness can entail the decrease of adhesion force value.

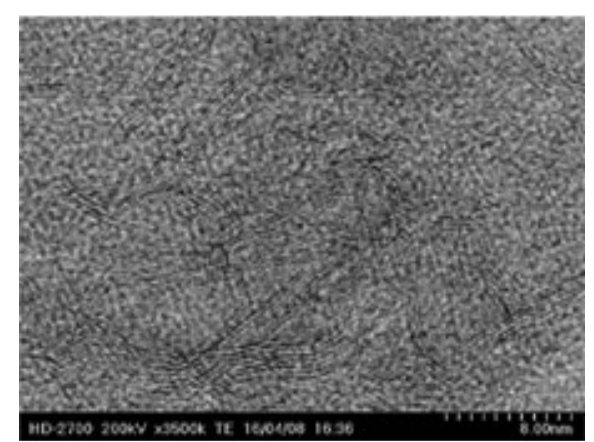

Figure 5. HR-STEM image of f-SWCNTs:P3OT nanocomposite

Figure 5 illustrates the HR-STEM image in which we can observe the presence of both individual carbon nanotubes and bundles. We can notice a high density of the f-SWCNTs bundles into the polymer matrix.

\section{Conclusions}

Two thin films were obtained by spin-coating technique using a pure $\mathrm{P} 3 \mathrm{OT}$ polymer and a mixture of P3OT as the host and $12 \%$ mass concentration functionalized single wall carbon nanotubes as the reinforcing phase. In order to examine the effect of the carbon nanotubes addition into the polymer matrix, AFM studies such as topography, roughness and force distance curves were performed.

The experimental results proved the noteworthy influence of the carbon nanotubes on both, morphological and mechanical properties of the analysed samples. The presence of the $\mathrm{f}$ SWCNTs into the polymer matrix leads to an increase of the surface roughness, while the adhesion force values are decreasing.

Also, with the carbon nanotubes addition into the host, the manner in which the adhesion values are distributed becomes more uniform.

\section{Acknowledgment}

This work was supported by Romanian Ministry of Education and Scientific Research, as well as by Executive Agency for Higher Education, Research, Development and Innovation Funding, under projects PCCA 2- nr. 66/2014 and PCCA 2-nr. 166/2012.

\section{References}

${ }^{1}$ C. Gerber, H.P. Lang, Nat. Nanotechnol. 1, 3-5 (2006)

2 Costantino Creton, Gwendal Josse, Rebecca Webber, and Dominique Hourdet, April, 10-13, (2006)

${ }^{3}$ Kevin Kendall, Kluwer

Academic / Plenum Publisher, New York (2001)

${ }^{4} \mathrm{~J}$. AL-Zanganawee, S. Iftimie, T. Mubarak, A. Radu, O. Brincoveanu, S. Antohe, M. Enachescu, Journal of Ovonic Research 2, 95 (2016)

${ }^{5}$ Vitali Brand, Christopher Bruner, Reinhold H. Dauskardt, Solar Energy Materials \& Solar Cells 99, 182-189, (2012)

${ }^{6}$ R. Phatak, T. Y. Tsui, and H. Aziz, Journal of Applied Physics, 111(5), 054512 (2012)

${ }^{7}$ Stephanie R. Dupont, MarkOliver, Frederik C. Krebs, Reinhold H. Dauskardt, Solar Energy Materials \& Solar Cells 97, 171-175, (2012)

${ }^{8}$ D. Qian, E. C. Dickey, R. Andrews, T. Rantell, Appl. Phys. Lett, 76, 2868-2870 (2000)

9 E. Kymakis, I. Alexandou, G.A.J. Amaratunga, Synthetic Metals, 127, 59-62 (2002)

${ }^{10}$ J-H, Du, J. Bai, H-M. Cheng, Polymer Letters 1 (5), 253-273 (2007)

${ }^{11}$ Marko Sedlac, BojanPodgornik, Joze Vizintin, Tribology International 48, 102-112, (2012)

${ }^{12}$ H. A. Mizes, K.G. Loh, R. J. D. Miller, S. K. Ahuja, and E. F. Grabowski, Applied Physics Letters 59 (22), 2901-2903 (1991) 